\title{
ENTORNOS VIRTUALES DE APRENDIZAJE Y DESARROLLO DE COMPETENCIAS DIGITALES EN LOS DOCENTES
}

\author{
Virtual environments of learning and development of digital competences in teachers
}

\author{
Yolanda LLamacponca Román 1, ${ }^{\text {: }}$ \\ ${ }^{1}$ Universidad Andina del Cusco, Cusco, Perú. \\ ”yllamacponca@uandina.edu.pe
}

\begin{abstract}
Resumen
El trabajo de investigación está encaminado a determinar la relación entre el uso del entorno virtual de aprendizaje y el desarrollo de las competencias digitales en los docentes de la región del Cusco, 2016. La calidad del entorno virtual de aprendizaje utilizado por los docentes de la región Cusco es muy bueno con el 77,6\% seguido por 20,6\% como bueno de igual modo el nivel de competencias digitales en los docentes de la región del Cusco se encuentran en el nivel avanzado con el 51,4\%, entonces los resultados demuestran que el uso de los entornos virtuales de aprendizaje por la presencia de diferentes recursos y herramientas digitales permite al docente desarrollar y elevar el nivel de las competencias digitales como en su desarrollo profesional, para la innovación mediante la transformación de las prácticas educativas con el apoyo de la TIC.

Palabras clave: Competencias digitales, entornos virtuales de aprendizaje.
\end{abstract}

\begin{abstract}
The research work is aimed at determining the relationship between the use of the virtual learning environment and the development of digital competences in teachers in the region of Cusco, 2016. The quality of the virtual learning environment used by teachers in the Cusco region is very good with $77.6 \%$ followed by $20.6 \%$ as well as equally the level of digital skills in the teachers of the Cusco region are found At the advanced level with $51.4 \%$, then the results show that the use of virtual learning environments by the presence of different digital resources and tools allows the teacher to develop and raise the level of digital skills as in their professional development, For innovation through the transformation of educational practices with the support of ICT.

Keywords: Digital competences, virtual learning environments.
\end{abstract}

Citar como: Llamacponca, Y.(2018). Entornos virtuales de aprendizaje y desarrollo de competencias digitales en los docentes. Rev Yachay, $7(1), 411-416$.

Recibido: 16-09-2018; Aceptado 07-12-2018

\section{Introducción}

En los últimos años la presencia de la tecnología ha revolucionado y reorganizado la manera en que vivimos, de cómo trabajamos, de cómo nos comunicados, de cómo aprendemos, los estudiantes son nativos digitales entrando en contacto con la tecnología a edades muy tempranas, aprender de manera activa y multimodal, invierten tiempo aprendiendo fuera de las aulas entre pares haciendo uso con diferentes herramientas a su alcance. La educación no puede enfrascarse en la reproducción de la información, la sociedad del conocimiento requiere de ciudadanos con la capacidad de aprender en la era donde se ofrece información exuberante, accesible y en un cambalache constante.

Según Gros (2004), los entornos virtuales son espacio diseñados con finalidades formativas, son ambientes de interacción social mediante el uso de la comunicación síncrona y asíncrona se promueve la participación activa de los estudiantes como actores, co-construyentes, pueden ser también diseñadores, productores de contenidos, reforzar enlaces. Los entornos virtuales de aprendizaje integran diferentes tecnologías y también enfoques pedagógicos múltiples; estas herramientas auxilian las múltiples funciones como la información, comunicación, colaboración, aprendizaje, gestión, etc. 
Los cursos virtuales para cumplir con su propósito de aprendizaje de calidad, deben de cumplir con ciertos protocolos didácticos y tecnológicos, proceso de virtualización que implica el asesoramiento, seguimiento de las tareas. Por ello se realiza una evaluación buscando la satisfacción total de los usuarios para ello se toman los siguientes criterios o dimensiones considerado por Santoveña S. (2010), la calidad general del entorno, la calidad didáctica y metodológica y la calidad técnica.

Entiéndase las competencias digitales como el conjunto de conocimiento, habilidades, actitudes, estrategias y concienciación que el uso de las TIC y de los medios digitales requieren para realizar tareas, resolver problemas, comunicar, gestionar la información, colaborar, crear y compartir contenidos y generar conocimiento de forma efectiva, eficaz, adecuada, critica, creativa, autónoma, flexible, ética, reflexiva para el trabajo, el ocio, la participación, el aprendizaje, la socialización, el consumo y el empoderamiento. Marco europeo de competencias digitales (2013).

La Comisión Europea dentro del proyecto de Marco Común de competencias digitales docentes. Describe las áreas de competencia como las herramientas a desarrollar en cada competencia.

La competencia de información; el docente identifica, localiza, almacena, organiza y analiza la información digital, evaluando su finalidad y relevancia, utiliza las herramientas de Explorer, Chrome, Firefox, Opera, Netscape, google académico, dialnet, lectores RSS, microblogging, blogs, poscasts, youtube, google drive, Dropbox, skydrive, evernote, simbaloo edu, etc.

La competencia de comunicación el docente participa, se comunica en entornos virtuales, forma parte de diferentes comunidades digitales y redes sociales. Utilizando las herramientas como Webex, Skype, google hangouts, Moodle, Edmodo, MOOC, webinars, streaming, Facebook, twitter, Tuenti, Badoo, blogs, microblogs, wikis, slideshare, picasa, flickr, scoop it., wordpress, blog, wiki, blogger, wikispaces, classroom, linkedln, leepass, mypermissions cleaner, etc.

En la competencia de creación de contenidos el docente debe crear y editar contenidos educativos nuevos integrando y reelaborando conocimientos y producciones artísticas, utilizándolas herramientas como Word, power point, mindomo, prezzy, sway, haiku deck, infographics toolbox de google, picmonkey, audacity, movie, jing, formulario de google, etc.

Competencia de seguridad consiste en la protección de los datos personales, respetar la privacidad de los demás, protegerse a sí mismo de amenazas y ciberacoso, protección de la identidad digital.

Competencia de resolución de problemas, es la capacidad para seleccionar, utilizar y combinar de forma pertinente, responsable y eficientemente las herramientas tecnológicas según el propósito o la necesidad, resolver problemas conceptuales a través de medio digitales, utilizar las tecnologías de forma creativa, resolver los problemas técnicos, actualizar su propia competencias y la de otros. Utilizando herramientas como Flash, Prezi, Coreldraw, PhotoShop, Gimp, Instagram, Access, MySQL, Dbase, ORACLE, Dreamweaver, Wordpress, otros.

En Actualmente en el Perú no se cuenta con un perfil de docente digital, como tampoco existe un diagnóstico real del nivel de competencias digitales, por ello surge la necesidad de realizar la presente investigación que permite realizar un diagnóstico de las competencias digitales de los docentes de EBR regular y que a partir de los resultados de esta investigación se puedan formular planes y programas de capacitación docentes en la región del Cusco utilizando entornos virtuales de aprendizaje que rompa fronteras y que abarque a más usuarios, donde cada uno pueda trabajar a su ritmo y estilo de aprendizaje y de manera mucho más económico. Por otra parte, los resultados de la presente investigación son importantes porque permitirán realizar otras investigaciones orientados a cambiar la realidad descrita.

\section{Materiales y Métodos}

Tipo y diseño de estudio: tipo sustantivo básico, no experimental transversal correlacional, puesto que se trata de medir y evaluar la relación de dos variables: nivel de satisfacción y desarrollo de competencias digitales en los docentes. El diseño es la transeccional correlacional porque se ha establecido la relación de asociación entre las variables de estudio, sin precisar el sentido de causalidad o pretender analizar relaciones causales

Población y muestra: La población estuvo constituida 1200 docentes, quienes laboraron en las instituciones educativas ubicados en 6 provincias en donde se ejecutaron los cursos de actualización en didáctica. La muestra es de 107 docentes que acceden a cursos virtuales de PerúEduca.

Rev. Yachay volumen (7) Número (1), enero-diciembre 2018 


\section{Técnicas e instrumentos}

Esta técnica permitió recoger y generar información primaria a través de un cuestionario virtual. Se utilizaron 02 instrumentos para el acopio de información, el instrumento $\mathrm{N}^{\circ} 01$ recoge información sobre la variable entornos virtual de aprendizaje con 36 items. El instrumento $n .^{\circ} 2$ para la variable competencia digital con 106 items.

\section{Resultados}

Los resultados obtenidos de la aplicación de los instrumentos se aprecian que de las ciento siete docentes que respondieron las encuestas el 1,9\% indican que es regular los entornos virtuales de aprendizaje, el 20,6\% bueno y el 77,6\% muy bueno. Que la gran mayoría percibe a los entornos virtuales como muy bueno, en cuanto a la competencia digital el $41,1 \%$ se encuentran en el nivel intermedio de las competencias digitales en los docentes de la instituciones educativas de la región Cusco, el 51,4\% se encuentran en el nivel avanzado y el 7,5\% en el nivel muy avanzado. El nivel de docentes se encuentra en el nivel avanzado. En lo que respecta a la general Existe una relación directa y significativa entre los entornos virtuales de aprendizaje y desarrollo de competencias digitales en los docentes de las instituciones educativas de la Región Cusco, 2016, con el nivel de significancia es al 5\% siendo $\alpha=0.05$ es decir un nivel de confianza del $95 \%$.

Considerando que el estadístico Tau b Kendall obtenido fue de 0,625 el cual indica la existencia de una correlación positiva moderada entre las variables de estudio y el valor de $\mathrm{p}=0,018$ es menor a 0,05 ; se concluye que existe una asociación entre los entornos virtuales de aprendizaje y desarrollo de competencias digitales en los docentes de las instituciones educativas de la Región Cusco, 2016.

\section{Discusión}

De la aplicación de los instrumentos de recojo de datos y el análisis estadístico se precisa que los entornos virtuales de aprendizaje en las instituciones educativas de educación básico regular de la región Cusco, se halla como muy bueno con el 77,6\% seguido por $20,6 \%$ como bueno.

En cuanto a las dimensiones estudiadas, se afirma que esta se halla en nivel de muy bueno con el 58.8\% la dimensión entorno general, la dimensión didáctica con el $63.6 \%$ en el nivel muy bueno, la dimensión técnica con $75.7 \%$ con muy bueno, lo que indica que el nivel de calidad de los entornos virtuales de aprendizaje de la plataforma PerúEduca es muy bueno.

Estos datos son corroborados con los hallazgos de Gámiz (2009), Aguilar (2014), e Infantas, Pareja Silva(2015) en las investigaciones realizadas sobre entornos virtuales de aprendizaje que revela que los EVA ayudan en la adquisición de competencias tecnológicas y profesionales como consecuencia del cumplimiento de indicadores de calidad como la metodológica empleada, la variedad de recursos y herramientas digitales y el diseño de la plataforma favoreciendo al estudiante la autorregulación, autonomía, autoaprendizaje y reflexión en los estudiantes generando cambios.

Así mismo Sonia Santoveña Casal menciona que los ambientes virtuales de calidad deben responder a requerimientos técnicos y metodológicos que satisfagan las necesidades de los usuarios. Los usuarios que acceden a cursos virtuales lo hacen si les proporcionan la posibilidad de consultar materiales didácticos de calidad, que les contribuyan conocimientos y no solo información, que cuente con medios de comunicación y tutoría virtualizada de calidad.

La plataforma de PerúEduca cuenta con los requerimientos técnicos y metodológicos como la calidad en el uso de las herramientas de comunicación, de estudio y de evaluación, la calidad técnica general como la estabilidad, la diversidad, la utilidad, la funcionalidad, cuenta con elementos multimedia, es de fácil acceso asimismo tiene calidad en el diseño.

La variable competencias digitales se aprecia que los docentes de la región del Cusco se encuentran en el nivel intermedio con el 51,1\% y avanzado $51.4 \%$, y en sus dimensiones estudiadas, los docentes de educación básica de la región Cusco se aprecia en la dimensión información en el nivel muy avanzado con 43\%, en la dimensión comunicación en nivel avanzado es del 65,4\%, en la dimensión creación de contenidos el mayor porcentaje es del $38.3 \%$ en el nivel intermedio, en la dimensión seguridad $51.4 \%$ en el nivel avanzado y en la dimensión resolución de problemas con el $42.1 \%$ en el nivel intermedio. Los docentes tiene un nivel avanzado luego de haber participado en los cursos virtuales que brindo PerúEduca.

Remitiéndonos estudios realizados por Esteve (2015). En la exploración de la competencia digital de los estudiantes universitarios de educación, poseen un nivel básico de competencia digital, especialmente en lo que respecta a las habilidades digitales básicas; no siendo 
así la adquisición de las habilidades didácticas que permitan utilizar las TIC en los procesos de enseñanza-aprendizaje, asimismo señala que no disponen de las suficientes estrategias para, a través de las TIC, seguir aprendiendo y formándose como profesionales.

Por otra parte es necesario replantear el sistema educativo para dar respuesta a tales retos, donde los maestros resultan ser pieza clave para el desarrollo de la competencia digital que permita trabajar en una sociedad en red e integrarlas en el proceso de enseñanza-aprendizaje, transformando las prácticas pedagógicas en el aula.

Los estudios realizados por Díaz (2009) en las competencias TIC y la integración de las tecnologías de la información y comunicación de los docentes, el nivel de competencias se concentra en el nivel medio con un $77.5 \%$.

Así mismo el Marco de Común de competencias digitales docentes (2013), UNESCO (2008), ISTE (2008) y MEN (2013) realizan una descripción de las competencias digitales mínimas de competencias, para usar recursos digitales en sus tareas docentes, introducir cambios metodológicos, analizados en cinco dimensiones información, comunicación, creación de contenidos, seguridad y resolución de problemas. Estas competencias son identificar, localizar, recuperar, almacenar, organizar y analizar la información digital, comunicar en entornos digitales, compartir recursos a través de herramientas en línea, conectar y colaborar con otros a través de herramientas digitales, interactuar y participar en comunidades y redes; crear y editar contenidos nuevos, integrar y reelaborar conocimientos; protección personal, protección de datos, protección de la identidad digital, uso de seguridad, tomar decisiones a la hora de elegir la herramienta digital apropiada, resolver problemas conceptuales, problemas técnicos.

La correlación entre las dimensiones entorno virtual de aprendizaje y competencias digitales se afirma que existe relación directa y significativa. Coincidiendo en la investigación de Mónica Aguilar (2014) que indica que las aulas virtuales influyen significativamente en el aprendizaje por competencias de los estudiantes del curso de internado estomatológico de la Facultad de Odontología de la Universidad de San Martín de Porras.

Así mismo UNESCO (2011) plantea una serie de estándares ligares a las competencias digitales que deben de poseer los docentes. En una sociedad cambiante, compleja, rica en información y basada en conocimiento, ello demanda que los docentes y estudiantes deben de adquirir un nivel aceptable para vivir, aprender y trabajar en el mundo actual, adquiriendo las capacidades necesarias.

\section{Conclusión}

Con la aplicación de los instrumentos de recojo de datos y el análisis estadístico se precisa que existe una relación directa y significativa entre los entornos virtuales de aprendizaje y desarrollo de competencias digitales en los docentes de las instituciones educativas de la Región Cusco, Considerando que el estadístico Tau b Kendall obtenido fue de 0,625 el cual indica la existencia de una correlación positiva moderada entre las variables de estudio concluyendo que existe una asociación entre las variables, estos resultados manifiestan que los docentes que hicieron uso de los EVA en PerúEduca desarrollaron competencias digitales, como consecuencia de la presencia de actividades, recursos y herramientas que demandan la necesidad de realizar las tareas, resolver problemas, comunicar y gestionar la información haciendo uso del conocimientos, habilidades, estrategias en el uso de las TIC y de los medios digitales.

Los entornos virtuales de aprendizaje en el Perú brotan como una alternativa viable flexible y eficaz para desarrollar programas educativos de calidad, como una manera para democratiza el acceso a una educación de calidad y una herramienta para viabilizar la educación permanente como se observa en la investigación.

En cuando a los entornos virtuales de aprendizaje en las instituciones educativas de educación básico regular de la región Cusco, se halla en el nivel muy bueno con el 77,6\%, lo que indica que el nivel de calidad de los entornos virtuales de aprendizaje de la plataforma PerúEduca es muy bueno, podemos derivar que la plataforma de PerúEduca cuenta con los requerimientos técnicos, metodológicos y psicopedagógicos, como consecuencia se convierte en un recurso innovador que permite en los docentes mejorar sus competencias profesionales y digitales.

\section{Recomendaciones}

Las instituciones educativas, la UGEL, DREC, municipios directores, APAFA, deben de realizar planes, programas que promuevan en docentes capacitaciones, utilizando las plataformas virtuales para una formación virtual de calidad, incluyendo un seguimiento, acompañamiento en el proceso de formación, considerando la creación de comunidades de aprendizaje, de investigación y de colaboración entre docentes en el contexto peruano. 
Potenciar el uso de los recursos y herramientas de los entornos virtuales de aprendizaje similares a PerúEduca que favorezcan la enseñanza y aprendizaje combinando con cursos b-learning.

Los docentes prepararse y desarrollar competencias digitales que permita la incorporación de las TIC al currículo y promueva estrategias metodológicas permitiendo el aprendizaje cooperativo haciendo uso de los entornos virtuales de aprendizaje.

\section{Referencia Bibliográfica}

Aguilar Valle, M. (2014), Influencia de las aulas virtuales en el aprendizaje por competencias de los estudiantes del curso internado estomatológico de la facultad de odontología de la universidad de San Martin de Porres. (Tesis doctoral, Universidad de San Martin de Porres, Lima, Pecú http://www.repositorioacademico.usmp.edu.pe/bitstream/usmp/1069/1/aguilar_vm.pdf

Díaz C, Ingrid (2009). Las competencias TIC y la integración de las tecnologías de la información y comunicación de los docentes de la Universidad Católica del Maule. (Tesis del grado de Magister, Universidad Católica del Maule, Santiago, Chile) Recuperado de http://repositorio.uchile.cl/tesis/uchile/2009/cs-diaz_i/pdfAmont/cs-diaz_i.pdf

DIGCOMP (2014). Marco europeo de competencias digitales. Recuperado de http://educalab.es/documents/10180/12809/MarcoComunCompeDigiDoceV2.pdf

Enlaces (2008) Estándares TIC para la formación inicial docente: Una propuesta en el contexto Chileno. Chile. Santiago de Chile, Chile, Ediciones LOM. Recuperado en http://historico.enlaces.cl/tp_enlaces/portales/tpe76eb4809f44/uploadImg/File/Competencias/Estandares\%20TIC\%20para $\% 20 F I D . p d f$

Gámiz Sánchez, V. (2009). Entornos virtuales para la formación práctica de estudiantes de Educación: Implementación, experimentación y evaluación de la plataforma AulaWeb. (Tesis doctoral, Universidad de Granada, Granada, España). Recuperado de http://hera.ugr.es/tesisugr/1850436x.pdf

Gros, B. (2004) La construcción del conocimiento en la red: límites y posibilidades. Teoría de la educación: Educación y cultura en la Sociedad de la información. Recuperado de www.usual.es/ teoriaeducacion/rev_numero_05/n4_gros.htm

Gros,B. y Silva J. (2005), La formación del profesorado como docentes en los espacios virtuales de aprendizaje. Revista Iberoamericana de Educación 36(I). Recuperado de www.campus-oei.org/revista/tec_edu32.tem

Gros. B. (2002), Constructivismo y diseños de entornos virtuales de aprendizaje. Revista de Educación 328, $225-247$.

IMCO Staff (2015), Reporte Global de Información Tecnológica 2015 vía WEF. Recuperado de http://imco.org.mx/banner_es/reporteglobal-de-informacion-tecnologica-2015-via-wef/

Infantes G., Pareja V. y Silva P. (2015) Uso educativo del entorno PerúEduca web. Estudio de caso de docentes de una institución educativa de la UGEL 05 de Lima Metropolitana. (Tesis del grado de Magister, Pontificia Universidad Católica del Perú, Lima, Perú) Recuperado http://tesis.pucp.edu.pe/repositorio/bitstream/handle/123456789/6780/INFANTES_PAREJA_SILVA_USO_EDUCATIV O.pdf? sequence $=1$

Unión Internacional de Telecomunicaciones (2015). Medición de la sociedad de la información. Recuperado de https://www.itu.int/en/ITU-D/Statistics/Documents/publications/misr2015/MISR2015-ES-S.pdf

Marco Europeo (2015). Competencias digitales dentro del Proyecto DIGCOMP. Recuperado de http://www.kzgunea.eus/competencias-digitales-con-kzgunea

Marcone S. (2013). Políticas Educativas y TIC en el Perú: apuesta, por la Calidad y la Inclusión, TIC para la inclusión y el Desarrollo Sostenible. Lima

Ministerio de Educación Nacional (2013). Competencias TIC para el desarrollo profesional docente. Recuperado de http://www.colombiaaprende.edu.co/html/micrositios/1752/articles-318264_recurso_tic.pdf

Ministerio de Educación (2012) Marco del buen desempeño docente, Lima, Perú: Autor.

Mishra, P., \& Koehler, M. J. (2006). Conocimiento Tecnológico disciplinar TPACK: presentación general de la teoría. Recuperado de https://issuu.com/huguibetoben/docs/si_ntesis_modelo_tpack

Organización para la Cooperación y el Desarrollo Económico (2010). Habilidades y competencias del siglo XXI para los aprendices del nuevo milenio en los países de la Organización para la Cooperación y el Desarrollo Económicos. Recuperado de http://recursostic.educacion.es/blogs/europa/media/blogs/europa/informes/Habilidades_y_competencias_siglo21_OCDE.p $\mathrm{df}$

Santoveña S. (2005), Criterios de calidad para la evaluación de los cursos virtuales. Publicación en línea. Granada (España) Año II N ${ }^{\circ}$ 4. Recuperado de http://tecnologiaedu.us.es/cuestionario/bibliovir/calidad.pdf 
Santoveña, S. (2010), Cuestionario de evaluación de la calidad de los cursos virtuales de la UNED. Revista de Educación a distancia. Número 25. Recuperado de http://www.um.es/ead/red/25/santovena.pdf

Santoveña. S. (2006), Metodología didáctica en los entornos virtuales de aprendizaje. Publicación en línea. Granada (España) No 3. Recuperado de http://www.ugr.es/ sevimeco/revistaeticanet/Numero3/Articulos/Formateados/metodologia_didactica.pdf

UNESCO (1998). La Educación Superior en el Siglo XXI. Visión y Acción. Documento de trabajo de la Conferencia Mundial sobre la Educación Superior, recuperado de http://www.unesco.org/education/educprog/wche/declaration_spa.htm

UNESCO (2008). "Estándares de competencia en TIC para docentes". Organización de las Naciones Unidas para la Educación, la Ciencia y la Cultura, París. Recuperado de http://eduteka.icesi.edu.co/articulos/EstandaresDocentesUnesco

Villar. G. (2007). La evaluación de un curso virtual. Propuesta de un modelo. Recuperado de www.oei.es/tic/villar.pdf
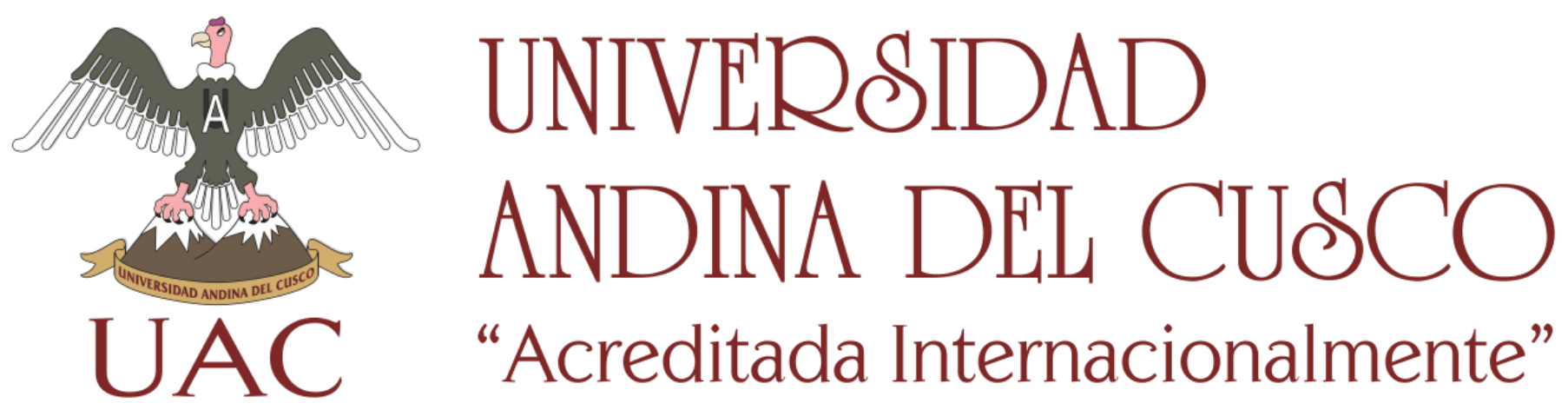